\title{
Three-Dimensional Simulations of Magnetized Thin Accretion Disks around Black Holes: Stress in the Plunging Region
}

\section{Citation}

Shafee, Rebecca, Jonathan C. McKinney, Ramesh Narayan, Alexander Tchekhovskoy, Charles F. Gammie, and Jeffrey E. McClintock. 2008. "Three-Dimensional Simulations of Magnetized Thin Accretion Disks Around Black Holes: Stress in the Plunging Region." The Astrophysical Journal 687 (1) (October 3): L25-L28. doi:10.1086/593148.

\section{Published Version}

doi:10.1086/593148

\section{Permanent link}

http://nrs.harvard.edu/urn-3:HUL.InstRepos:27804416

\section{Terms of Use}

This article was downloaded from Harvard University's DASH repository, and is made available under the terms and conditions applicable to Other Posted Material, as set forth at http:// nrs.harvard.edu/urn-3:HUL.InstRepos:dash.current.terms-of-use\#LAA

\section{Share Your Story}

The Harvard community has made this article openly available.

Please share how this access benefits you. Submit a story.

Accessibility 


\title{
THREE-DIMENSIONAL SIMULATIONS OF MAGNETIZED THIN ACCRETION DISKS AROUND BLACK HOLES: STRESS IN THE PLUNGING REGION
}

\author{
Rebecca Shafee, ${ }^{1}$ Jonathan C. McKinney, ${ }^{2}$ Ramesh Narayan, ${ }^{3}$ Alexander Tchekhovskoy, ${ }^{3}$ \\ Charles F. Gammie, ${ }^{4}$ and JefFrey E. McClintock ${ }^{3}$ \\ Received 2008 August 10; accepted 2008 September 12; published 2008 October 3
}

\begin{abstract}
We describe three-dimensional general relativistic magnetohydrodynamic simulations of a geometrically thin accretion disk around a nonspinning black hole. The disk has a thickness $h / r \sim 0.05-0.1$ over the radial range $(2-20) G M / c^{2}$. In steady state, the specific angular momentum profile of the inflowing magnetized gas deviates by less than $2 \%$ from that of the standard thin disk model of Novikov and Thorne. Also, the magnetic torque at the radius of the innermost stable circular orbit (ISCO) is only 2\% of the inward flux of angular momentum at this radius. Both results indicate that magnetic coupling across the ISCO is relatively unimportant for geometrically thin disks.
\end{abstract}

Subject headings: accretion, accretion disks — binaries: close — black hole physics — X-rays: stars

\section{INTRODUCTION}

The recent development of general relativistic magnetohydrodynamic (GRMHD) codes (e.g., Gammie et al. 2003; De Villiers \& Hawley 2003) has finally allowed realistic numerical simulations of magnetized accretion disks around black holes (BHs). This has led to a better understanding of the inner regions of these disks (e.g., Krolik et al. 2005) and of their role in launching relativistic jets (e.g., McKinney 2006). One of the interesting new results is the recognition that magnetic fields alter the structure of the accretion flow near and inside the innermost stable circular orbit (ISCO). This may result in large deviations from the traditional picture of a vanishing torque at the ISCO (Krolik 1999; Gammie 1999; Krolik et al. 2005).

Paczyński (2000) and Afshordi \& Paczyński (2003) suggested that the zero-torque condition is likely to be a good approximation for geometrically thin disks. This was confirmed by Shafee et al. (2008) who, using a global height-integrated model, showed that modifications to the stress profile are negligibly small for disk thicknesses $h$ less than about a tenth of the local radius $r$. Their work was, however, based on a hydrodynamic model with $\alpha$-viscosity and did not explicitly include magnetic fields.

Numerical MHD simulations by Krolik \& Hawley (2002) using a pseudo-Newtonian potential, and by Krolik et al. (2005) using a GRMHD code, indicated that magnetic torques are indeed important inside the ISCO. In particular, these authors found no evidence for a "stress edge," leading them to argue that simple disk models based on the zero-torque condition (e.g., Shakura \& Sunyaev 1973; Novikov \& Thorne 1973, hereafter NT73) may be seriously wrong. If true this would undermine recent efforts to estimate the spin parameters of BHs using the NT73 model (Shafee et al. 2006; McClintock et al. 2006; Davis et al. 2006; Liu et al. 2008).

The MHD simulations carried out so far have considered

\footnotetext{
${ }^{1}$ Harvard University, Department of Physics, 17 Oxford Street, Cambridge, MA 02138.

${ }^{2}$ Chandra Fellow; Kavli Institute for Particle Astrophysics and Cosmology, Stanford University, CA 94309.

${ }^{3}$ Harvard-Smithsonian Center for Astrophysics, 60 Garden Street, Cambridge, MA 02138.

${ }^{4}$ Center for Theoretical Astrophysics, University of Illinois, Urbana-Champaign, IL 61801.
}

nonradiating accretion flows that are geometrically rather thick. The one exception is the recent work of Reynolds \& Fabian (2008) who considered a thin disk with $h / r \sim 0.05$ in a pseudoNewtonian potential. In this Letter, we describe global 3D GRMHD simulations of a thin disk $(h / r \sim 0.05)$ around a nonspinning BH and compare our simulated model with the NT73 model.

\section{NUMERICAL MODEL}

We use units with $G=c=1$, e.g., the horizon is at $r=$ $2 M$ and the ISCO is at $6 M$. We report results in Boyer-Lindquist (BL) coordinates $(t, r, \theta, \phi)$, referred to as the coordinate frame. In the expressions below $g$ refers to the determinant of the metric. The fluid 4-velocity and the magnetic field 4-vector (see, e.g., Anile 1989 for definitions) are given by $u^{\mu}$ and $b^{\mu}$, and the rest-mass density, internal energy density, thermal pressure, and magnetic pressure as measured in the fluid comoving frame are $\rho, u_{g}, p=(\gamma-1) u_{g}$ with adiabatic index $\gamma=4 / 3$, and $p_{b}=b^{2} / 2$. The total pressure is $p_{\text {tot }}=p+p_{b}$.

Simulations were performed using a 3D GRMHD code HARM (Gammie et al. 2003) in Kerr-Schild coordinates using the interpolation scheme described by McKinney (2006) inversion scheme described by Noble et al. (2006) and Mignone \& McKinney (2007) and other advances described by Tchekhovskoy et al. (2007). For our fiducial model we used a 3D grid with resolution $512 \times 128 \times 32$ corresponding to (1) 512 cells in $r$, logarithmically spaced from $r=1.8 M$ to $50 M$ with "outflow" boundary conditions; (2) 128 cells in $\theta$ going from 0 to $\pi$, nonuniformly spaced so that roughly half the cells are concentrated in the disk; ${ }^{5}$ (3) 32 cells in $\phi$, uniformly spaced from 0 to $\pi / 4$. Cells at the disk equator had physical sizes roughly in the ratio of $2: 1: 7$ in $d r, r d \theta, r \sin (\theta) d \phi$, which ensured that the turbulence was roughly isotropic in Cartesian coordinates, optimal for accurately resolving the magnetorotational instability (MRI). To test convergence we also used resolutions of $256 \times 64 \times 16,256 \times 64 \times 32$, and $256 \times$ $64 \times 64$. We also carried out $2 \mathrm{D}$ simulations with resolution of up to $2048 \times 256 \times 1$, but we do not discuss these results

\footnotetext{
${ }^{5}$ We used a grid given by $\theta\left(x^{(2)}\right)=\left[h\left(2 x^{(2)}-1\right)+(1-h)\left(2 x^{(2)}-1\right)^{7}+\right.$ 1] $\pi / 2$ for code coordinate $x^{(2)}$ with $h=0.15$, giving roughly 6 times more angular resolution compared to eq. (8) with $h=0.3$ in McKinney \& Gammie (2004).
} 
because turbulence decayed on an orbital time-scale (i.e., Cowling's antidynamo theorem holds).

We began the simulation with an equilibrium torus (Chakrabarti 1985; De Villiers \& Hawley 2003) with inner edge at $r=20 M$ and pressure maximum at $r=35 M$ and adjusted the model parameters so the torus had $h / r=0.1$ at $35 M$. We found that placing the torus at a smaller radius led to results too sensitive to the initial mass distribution. We define $h / r$ to be the density-weighted root mean square angular thickness of the disk at any given $r$, i.e.,

$$
\left(\frac{h}{r}\right)_{r}=\Delta \theta_{\mathrm{rms}}=\left[\frac{\iint(\Delta \theta)^{2} \rho(r, \theta, \phi) \sqrt{-g} d \theta d \phi}{\iint \rho(r, \theta, \phi) \sqrt{-g} d \theta d \phi}\right]^{1 / 2},
$$

or written as $\Delta \theta_{\text {rms }}=\left\langle\Delta \theta^{2}\right\rangle^{1 / 2}$ with $\Delta \theta \equiv \theta-\langle\theta\rangle$. We also consider the mean density-weighted thickness: $h / r=\Delta \theta_{\mathrm{abs}}=$ $\langle|\Delta \theta|\rangle$.

We embedded the torus with a weak magnetic field with $\beta \equiv p / p_{b} \sim 100$. The initial field consisted of two poloidal loops centered at $r=28 M$ and $38 M$ to model a disorganized field with no net flux. (During the simulation, there is no organized flux threading the disk but some organized flux threads the BH.) The field strength was randomly perturbed by $50 \%$ to seed the MRI instability. Recent GRMHD simulations (McKinney \& Narayan 2007a, 2007b; Beckwith et al. 2008a) indicate that the results for the disk (but not the jet) should be roughly independent of the initial field geometry. The MRI is initially resolved with much of the torus having 10 cells per wavelength of the fastest growing MRI mode.

In order to keep the accretion disk thin, an ad hoc cooling function was added to the energy-momentum equations as a covariant source term $\left(-u^{\mu} d u_{g} / d \tau\right)$ with

$$
\frac{d u_{g}}{d \tau}=-\frac{u_{g}-u_{\mathrm{eq}}}{\tau_{\text {cool }}}
$$

where $\tau$ is the fluid proper time. The gas cooling time $\left(\tau_{\text {cool }}\right)$ was set to $2 \pi / \Omega_{\mathrm{K}}$, where $\Omega_{\mathrm{K}}=(r / M)^{-3 / 2} M$ is the Keplerian frequency. Thus the gas was driven toward $u_{\text {eq }}$, which we defined as that value of $u_{g}$ for which the specific entropy of the gas would be equal to the constant specific entropy (e.g., $\left.p / \rho^{\gamma}\right)$ of the initial solution.

The simulation ran for a time of $10000 M$, corresponding to 108 orbits at the ISCO $\left(r_{\text {ISCO }}=6 M\right)$ and 18 orbits at the initial inner edge of the torus. The results reported here correspond to averages computed over the period $7000 M-10000 M$, when the accretion flow had reached a quasi-steady state inside a radius of about $10 \mathrm{M}$. Figure 1 shows that the simulated disk had a thickness (given by eq. [1]) of $h / r \sim 0.06-0.10$ over the radius range of interest. The mean absolute thickness $\Delta \theta_{\text {abs }}$ was smaller: $\sim 0.04-0.07$. At the ISCO, the two definitions of thickness gave $h / r \sim 0.08,0.06$, respectively.

\section{RESULTS}

The flux of mass and specific angular momentum are given by

$$
\begin{aligned}
\dot{M}(r, t) & =-\iint \rho u^{r} \sqrt{-g} d \theta d \phi, \\
\frac{\dot{L}(r, t)}{\dot{M}(r, t)} & =-\frac{1}{\dot{M}(r, t)} \iint\left(T_{\phi, \text { in }}^{r}-T_{\phi, \text { out }}^{r}\right) \sqrt{-g} d \theta d \phi \\
& \equiv \bar{\ell}_{\text {in }}(r, t)-\bar{\ell}_{\text {out }}(r, t),
\end{aligned}
$$

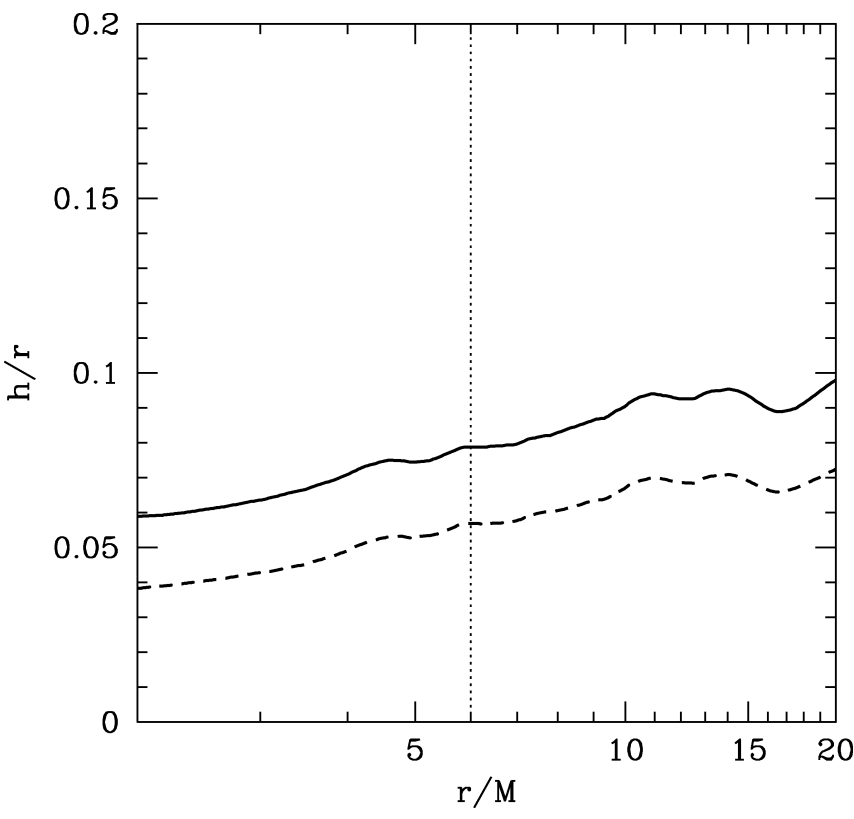

FIG. 1.-Variation of the root mean square disk thickness $h / r=\Delta \theta_{\text {rms }}$ (eq. [1]) as a function of radius $r$ (solid line). Simulation results were averaged over the time interval $7000 M-10000 M$. Also shown is the mean absolute thickness $\Delta \theta_{\text {abs }}$ (dashed line). The vertical dotted line corresponds to the position of the ISCO. The initial torus had its inner edge at $r_{\text {in }}=20 M$.

respectively, where $T_{\phi}^{r}$ is the $r$ - $\phi$ component of the stress-energy tensor, and

$$
T_{\phi, \text { in }}^{r}=\left(\rho+u_{g}+p+b^{2}\right) u^{r} u_{\phi}, \quad T_{\phi, \text { out }}^{r}=b^{r} b_{\phi} .
$$

The specific energy flux $\dot{E} / \dot{M}$ is found by replacing $T_{\phi}^{r}$ with $T_{t}^{r}$ in the above equations. In equation (5), the term $\ell_{\text {in }}$ represents the specific angular momentum advected inward with the accretion flow. Similarly, $\bar{\ell}_{\text {out }}$ represents outflow of specific angular momentum as a result of the magnetic shear stress. In steady state, each of these terms is independent of $t$, and their sum is independent of both $r$ and $t$.

Figure 2 compares the time-averaged profile of $\bar{\ell}_{\text {in }}$ from the simulation, averaged over an angular range $\delta \theta= \pm 0.2$ around the disk midplane ( 2 to 3 density scale heights), with that predicted by NT73. In the latter, $\ell_{\text {in }}$ is equal to the Keplerian specific angular momentum for all radii down to the ISCO; inside the ISCO, $\bar{\ell}_{\text {in }}$ is taken to be constant since, by assumption, no angular momentum is removed from the gas in the plunging region. The $\bar{\ell}_{\text {in }}$ profile from the simulation shows modest deviations from this idealized profile: (1) $\bar{\ell}_{\text {in }}$ is slightly sub-Keplerian outside the ISCO; (2) $\ell_{\text {in }}$ continues to decrease for a range of $r$ inside the ISCO; (3) $\ell_{\text {in }}$ becomes essentially independent of $r$ close to the horizon.

The surprising feature of Figure 2 is that the two curves are so close to each other inside the ISCO. While it is true that the profile of $\bar{\ell}_{\text {in }}$ from the simulation drops inside the ISCO to a value of $3.39 M$, this value is only $2.0 \%$ less than the NT73 value of $3.464 M$. Thus, the magnetic coupling that technically operates inside $r_{\text {Isco }}$ does not have much real effect on the accreting gas. At the ISCO $\ell_{\text {out }}=0.075 M$, which is $\sim 2.2 \%$ of $\ell_{\text {in. }}$. Further, $\dot{E} / \dot{M}$ at the horizon is 0.940 , which is only slightly different from the NT73 value of 0.9428 , showing that the accretion efficiency is not enhanced by magnetic fields. For two-dimensional simulations of thicker disks with $h / r \sim 0.3$, McKinney \& Gammie (2004) found that $\dot{L} / \dot{M}=3.068$ and 


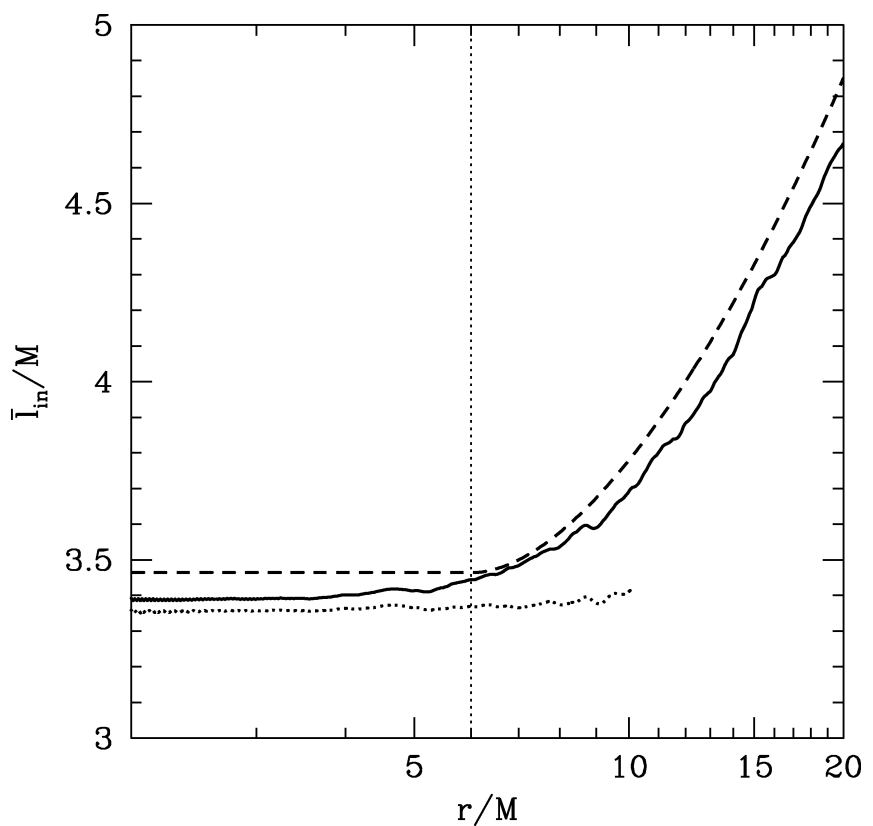

FIG. 2.-Time-averaged profile of the specific angular momentum $\bar{\ell}_{\text {in }}$ of the inflowing magnetized gas (solid line), compared to the idealized profile assumed in the NT73 disk model (dashed line). The horizontal dotted curve shows the net flux of angular momentum $\bar{\ell}_{\text {in }}-\bar{\ell}_{\text {out }}$. This is independent of $r$ up to $\sim 10 M$, indicating the simulation has achieved steady state well beyond the ISCO.

$\dot{E} / \dot{M}=0.950$. It appears that $\dot{L} / \dot{M}$ deviates from the NT73 value roughly proportional to $h / r$ and so thinner disks should be even closer to NT73. These results are converged since, between a resolution of $256 \times 64 \times 32$ and $512 \times 128 \times$ 32 , the value of $\dot{L} / \dot{M}$ changes by less than $0.2 \%$ and $\dot{E} / \dot{M}$ changes by less than $0.5 \%$.

Figure 3 shows the time-average of the normalized shear stress

$$
\tilde{W} \equiv 10^{3} \iint T_{\hat{\phi} \hat{r}} \sqrt{-g} d \theta d \phi /(2 \pi r \dot{M})
$$

where $W \equiv 10^{-3} \tilde{W} \dot{M}$ as in equation (5.6.1b) in NT73 and $T_{\hat{\phi} \hat{r}}$ is the orthonormal stress-energy tensor components in the comoving frame. We restrict the integral to fluid with $u_{t}(\rho+$ $\left.u+p+b^{2}\right) / \rho>-1$, i.e., only gravitationally bound fluid (no disk wind); this lowers the value of $W$ near the horizon by $50 \%$. Figure 3 also shows the space-time averaged viscosity parameter $\alpha \equiv T_{\hat{\phi} \hat{r}} / p_{\text {tot }}$, which is roughly $0.1-0.15$ outside the ISCO. Between the ISCO and $r=3 M, \alpha$ rises to 0.6 and finally drops to $\sim 0$ near the horizon. There are nonvanishing stresses inside the ISCO and the "stress edge" is near the horizon. These stresses appear due to organized magnetic flux, but evidently do not cause significant transport of angular momentum (Fig. 2) and are not expected to be a significant source of dissipation and radiation.

Another way of evaluating the degree of coupling is to consider, by analogy with steady state flows, the locations of "critical points," defined as the radii at which various volumeaveraged outgoing characteristic speeds vanish in the coordinate frame. For our GRMHD simulation, the fast magnetosonic radius is located at $r_{\text {fast }} \sim 5.1 M$ showing that, even for this relatively thin accretion disk, magnetic fields do enhance the communication between the disk and the plunging

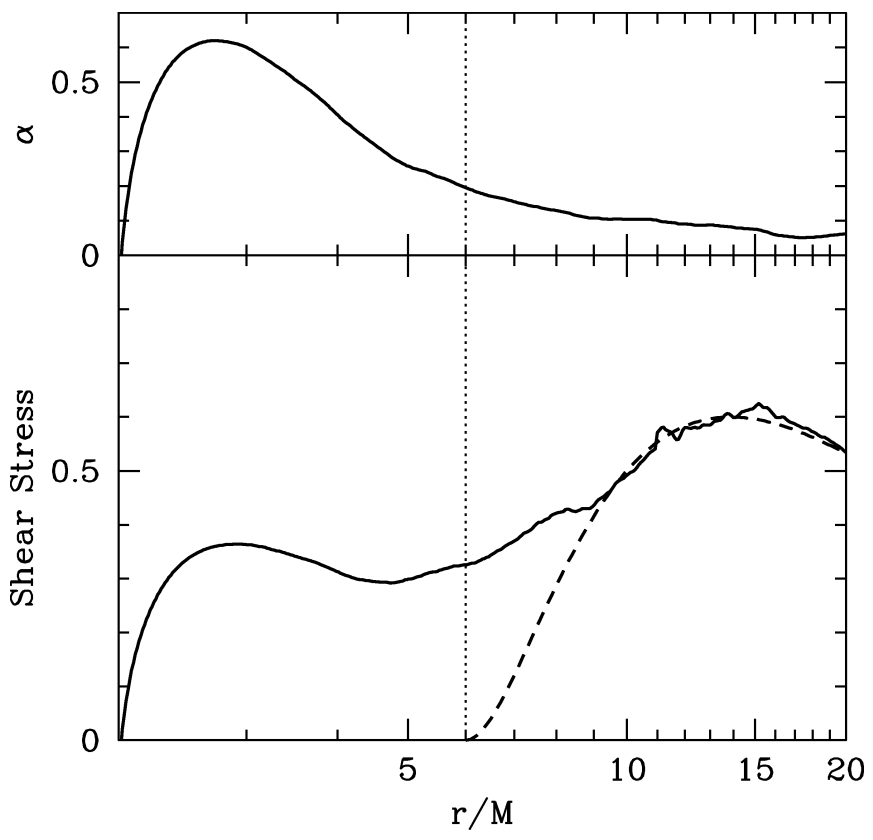

FIG. 3.-Bottom panel shows time-averaged profile of the fluid shear stress (solid line) compared to the NT73 stress (dashed line). Top panel shows $\alpha$ viscosity coefficient (solid line). The nonzero stress in the plunging region causes only a minor deviation from the NT73 result for the flux of specific angular momentum, as seen in Fig. 2.

region. On the other hand, the Alfvén critical point is much further out at 8.0M. Since Alfvén waves play an important role in angular momentum transport, this suggests that the shear coupling via magnetic fields is weak, unlike the suggestion of Krolik (1999) and Gammie (1999).

For fitting disk models to observations the most useful quantity is the rate of dissipation of energy as a function of radius, since this is what determines the radiation emitted by the accretion disk. As a simple estimate, consider the standard theory of viscous disks in which the energy dissipation rate is equal to the local shear stress multiplied by $r d \Omega_{\mathrm{K}} / d r$ (this is not necessarily valid for an MHD fluid, and is even less valid in the plunging region). In contrast to the standard model, which assumes a vanishing torque at the ISCO, we find $\bar{\ell}_{\text {out }}=$ $0.022 \bar{\ell}_{\text {in }}$ at $r=r_{\text {Isco. }}$. This modification to the inner boundary condition will cause the luminosity of the disk outside the ISCO to increase by about $4 \%$.

Figure 4 shows one measure of the magnitude of turbulent fluctuations,

$$
\xi_{\text {turb }}=\frac{\sqrt{\left\langle\left(v^{r}\right)^{2}\right\rangle-\left\langle v^{r}\right\rangle^{2}}}{\left\langle v^{r}\right\rangle},
$$

time- and angle-averaged as for Figure 2. We see that the gas at radii $\geq 10 M$ is highly turbulent due to the MRI. However, the gas flow is fairly smooth at smaller radii, suggesting that there is little dissipation of turbulent kinetic energy inside the ISCO.

\section{CONCLUSION}

For a geometrically thin accretion disk with $h / r \sim$ 0.05-0.1 (Fig. 1) around a nonspinning BH, we find that the specific angular momentum profile $\bar{\ell}_{\text {in }}(r)$ of the accreting magnetized gas is quite similar to that assumed in the idealized model of NT73 (Fig. 2). Specifically, $\bar{\ell}_{\text {in }}$ is slightly smaller 


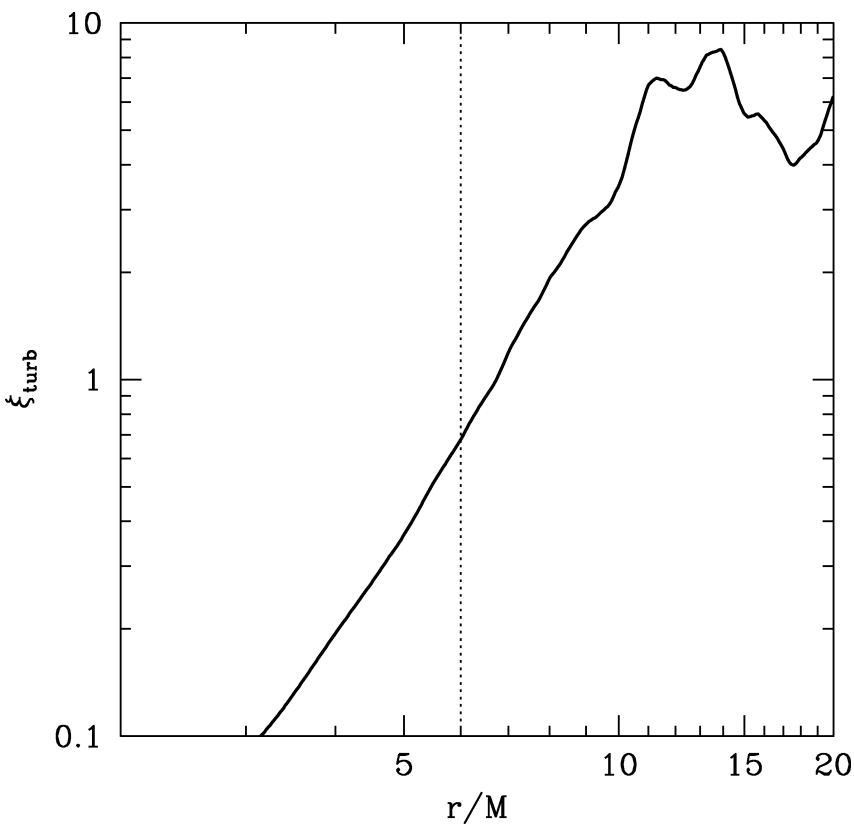

Fig. 4.-Time-averaged profile of $\xi_{\text {turb }}$, which measures the relative magnitude of turbulent fluctuations in the accreting gas. The fluid becomes mostly laminar inside the ISCO.

than, but nearly equal to, the Keplerian value of $\bar{\ell}$ for radii outside the ISCO, and is almost independent of $r$ inside the ISCO. At the $\mathrm{BH}$ horizon, the value of $\bar{\ell}_{\text {in }}$ deviates from the NT73 value by only $2.0 \%$.

This result suggests that the NT73 model is a good approximation for thin disks. However, fast magnetosonic waves from radii as small as $5.1 \mathrm{M}$ can escape the plunging region and communicate magnetic stresses between the disk and the plunging regions, which means that the zero-torque condition assumed in the NT73 model is not perfectly valid. Nevertheless, the normalized outward flux of angular_momentum $\bar{\ell}_{\text {out }}$ at the ISCO is only $\sim 2.2 \%$ of the inward flux $\bar{\ell}_{i n}$. Thus, the magnetic coupling across the ISCO is quantitatively weak. As a result, we estimate the luminosity of the disk to be enhanced by no more than a few percent.

We find that turbulent activity is pronounced at radii beyond about $10 M$, and that the flow is nearly laminar inside the ISCO. This suggests that the bulk of the dissipation occurs outside the ISCO in the disk proper, not in the plunging region. In our next paper we plan to discuss the energy dissipation profile of 3D GRMHD simulations, which is ultimately what determines the observed radiation. Future studies should also investigate the dependence of these results on $h / r$, magnetic field geometry, black hole spin (e.g., Gammie et al. 2004), cooling, resistivity and viscosity, and mass distribution.

The results reported here are in qualitative agreement with those obtained by Reynolds \& Fabian (2008). They carried out a 3D nonrelativistic MHD simulation in a pseudo-Newtonian potential, whereas ours is a fully relativistic GRMHD simulation. Also, their initial torus had its inner radius at the ISCO, $r_{\text {in }}=6 M$, whereas our torus initially had $r_{\text {in }}=20 M$. This might explain some differences in our results, e.g., their turbulence edge is at $5.5 \mathrm{M}$ whereas ours is at $6.8 \mathrm{M}$ (compare their Fig. 3 with our Fig. 4). Indeed, we find that our results are sensitive to the initial conditions if we place the inner edge of the torus much inside $r_{\text {in }} \sim 20 M$. Despite these differences the two simulations agree on the main result, viz., for a geometrically thin disk, the ISCO does behave like a physical boundary separating the disk proper from the plunging region. Beckwith et al. (2008b) suggest a larger deviation from NT73, but they considered a torus with a larger value of $h / r$ and did not use a self-consistent dissipation model. A study of the actual dissipation in the simulation, the goal of our next paper, will hopefully resolve these differences.

We thank Niayesh Afshordi for stimulating discussions and useful suggestions. The simulations described in this paper were run on the BlueGene/L system at the Harvard SEAS CyberInfrastructures Lab. This work was supported in part by NASA grant NNH07ZDA001N and NSF grant AST 08-05832. J. C. M. was supported by NASA's Chandra Postdoctoral Fellowship PF7-80048.

\section{REFERENCES}

Afshordi, N., \& Paczyński, B. 2003, ApJ, 592, 354

Anile, A. M. 1989, Relativistic Fluids and Magneto-Fluids (New York: Cambridge Univ. Press)

Beckwith, K., Hawley, J. F., \& Krolik, J. H. 2008a, ApJ, 678, 1180 2008b, preprint (arXiv:0801.2974)

Chakrabarti, S. K. 1985, ApJ, 288, 1

Davis, S. W., Done, C., \& Blaes, O. M. 2006, ApJ, 647, 525

De Villiers, J.-P., \& Hawley, J. F. 2003, ApJ, 589, 458

Gammie, C. F. 1999, ApJ, 522, L57

Gammie, C. F., McKinney, J. C., \& Tóth,G. 2003, ApJ, 589, 444

Gammie, C. F., Shapiro, S. L., \& McKinney, J. C. 2004, ApJ, 602, 312

Krolik, J. H. 1999, ApJ, 515, L73

Krolik, J. H., \& Hawley, J. F. 2002, ApJ, 573, 754

Krolik, J. H., Hawley, J. F., \& Hirose, S. 2005, ApJ, 622, 1008

Liu, J., McClintock, J. E., Narayan, R., Davis, S. W., \& Orosz, J. A. 2008, ApJ, 679, L37

McClintock, J. E., Shafee, R., Narayan, R., Remillard, R. A., Davis, S. W., \& Li, L.-X. 2006, ApJ, 652, 518
McKinney, J. C. 2006, MNRAS, 367, 1797

McKinney, J. C., \& Gammie, C. F. 2004, ApJ, 611, 977

McKinney, J. C., \& Narayan, R. 2007a, MNRAS, 375, 513

. 2007b, MNRAS, 375, 531

Mignone, A., \& McKinney, J. C. 2007, MNRAS, 378, 1118

Noble, S. C., Gammie, C. F., McKinney, J. C., \& Del Zanna, L. 2006, ApJ, 641,626

Novikov, I. D., \& Thorne, K. S. 1973, in Black Holes, ed. C. DeWitt \& B. S. DeWitt (New York: Gordon and Breach), 343

Paczyński, B. 2000, preprint (astro-ph/0004129)

Reynolds, C. S., \& Fabian, A. C. 2008, ApJ, 675, 1048

Shafee, R., McClintock, J. E., Narayan, R., Davis, S. W., Li, L.-X., \& Remillard, R. A. 2006, ApJ, 636, L113

Shafee, R., Narayan, R., \& McClintock, J. E. 2008, ApJ, 676, 549

Shakura, N. I., \& Sunyaev, R. A. 1973, A\&A, 24, 337

Tchekhovskoy, A., McKinney, J. C., \& Narayan, R. 2007, MNRAS, 379, 469 